Artículo

\title{
Comportamiento agronómico del garbanzo en el sector de El Limoncito, Ecuador
}

\author{
Ángel Bernardo Llerena Hidalgo ${ }^{\S}$ \\ Departamento de Agropecuaria-Facultad de Educación Técnica para el Desarrollo-Universidad Católica de \\ Santiago de Guayaquil. Guayaquil, Ecuador.

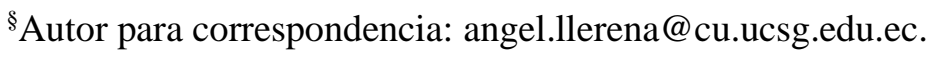

\section{Resumen}

En el presente estudio se evaluó el comportamiento agronómico del garbanzo tropicalizado de ascendencia peruana a diferentes distancias de siembra, en las condiciones edafoclimáticas de la zona de El Limoncito, provincia de Santa Elena, Ecuador. El diseño experimental aplicado fue el de diseño de bloques completamente al azar (DBCA) donde se estudiaron cinco tratamientos con diferentes distanciamientos de siembra los cuales fueron T1 $(0.1 \times 0.5 \mathrm{~m}), \mathrm{T} 2(0.2 \times 0.5 \mathrm{~m}), \mathrm{T} 3$ $(0.3 \times 0.5 \mathrm{~m}), \mathrm{T} 4(0.4 \times 0.5 \mathrm{~m})$ y $\mathrm{T} 5(0.5 \times 0.5 \mathrm{~m})$. En cada tratamiento se analizaron las variables de altura de la planta, número de flores, numero de ramas, numero de vainas y rendimiento. Los resultados obtenidos muestran que el T2 con distanciamiento de 0.2 x $0.5 \mathrm{~m}$ da resultados óptimos en cuanto a altura de la planta, número de flores, número de ramas, número de vainas y rendimiento.

Palabras clave: adaptabilidad, edafoclimatología, garbanzo.

Recibido: mayo de 2021

Aceptado: julio de 2021 


\section{Introducción}

El garbanzo es el tercer cultivo de leguminosas alimenticias más importante del mundo (Heidarvand y Maali-Amiri, 2013). El sur de Asia es el principal productor de garbanzos, que aporta aproximadamente las tres cuartas partes de la producción mundial de garbanzos. La producción mundial de garbanzo en el periodo 2010-2018, registró un crecimiento de 3.9 millones toneladas (Mt), lo que significó un considerable incremento para dicho periodo (FAO, 2020). Comercialmente se producen dos genotipos de garbanzo, desi y kabuli. El genotipo kabuli tiene semillas más grandes, redondas y de color crema, mientras el genotipo desi tienen semillas relativamente más pequeñas, de forma angular y de color oscuro (Nisa et al., 2020).

El garbanzo es un grano de alto poder nutricional, el contenido de proteína para los garbanzos desi y kabuli es de 16.7 a $30.6 \%$ y 12.6 a $29 \%$ respectivamente (Wood y Grusak, 2007). Lo mencionado indica que el cultivo de garbanzo es parte integral de la estabilización productiva de granos ricos en proteínas, así como en otros sistemas agrícolas en general (Kazydub et al., 2019).

Además de su importancia nutricional el cultivo de garbanzo mejora la fertilidad de los suelos agrícolas manteniendo la productividad de los sistemas de cultivo debido a su capacidad para fijar nitrógeno atmosférico (Sharma et al., 2018). En promedio, las cantidades estimadas de N fijadas por los garbanzos en condiciones de precipitación regular y estrés por sequía son $60 \mathrm{~kg} \mathrm{ha}^{-1}$ y 19$24 \mathrm{~kg} \mathrm{ha}^{-1}$, respectivamente (Carranca et al., 1999; Abi-Ghanem et al., 2012).

El garbanzo es bastante resistente a las sequías, pero no se adapta a condiciones de alta humedad. Puede ser cultivado de secano en un clima fresco y seco en las regiones semiáridas y también, pero con mejores rendimientos, en riego (Saluzzo, 2010; Coirini y Nolasco, 2016). Además de una preparación adecuada del campo, el uso de semillas de alto rendimiento, la fertilización y otras prácticas culturales, la programación y el riego también son parámetros importantes en el cultivo del garbanzo (Kirnak et al., 2017).

Durante las últimas décadas, debido al aumento de la demanda de alimentos, la creciente población mundial depende en gran medida de la conservación y el uso de los recursos fitogenéticos que quedan en el mundo (FAO, 1997; Sharma y Johnson, 2017). Estos deben ser estudiados y ser seleccionados de acuerdo con las variabilidades fenotípicas y genotípicas de los caracteres agronómicos esperados de un cultivo de buena producción (Arora, 1991).

Por lo tanto, es necesario la constante investigación de diferentes variedades de cultivos con el objetivo de disponer conocimientos de las variedades más optimas que puedan contrarrestar o tolerar los diferentes factores bióticos y abióticos que limitan la producción de los cultivos (Shagarodsky et al., 2001; Fierros et al., 2011).

La adaptabilidad implica una propiedad por el cual los órganos capacitados de una planta sobreviven y se reproducen en ambientes fluctuantes, por lo que es una habilidad genética que resulta de estabilizar las interacciones con el ambiente, por medio de relaciones genéticas y fisiológicas de los organismos (Anderson et al., 2011). 
En Ecuador antiguamente se sembraba garbanzo en la zona de la sierra perteneciente al sector de Riobamba de la provincia de Chimborazo. Lamentablemente por el ciclo vegetativo de seis meses de este cultivo las plantaciones fueron afectadas por plagas y enfermedades diezmaron prácticamente la utilidad a los productores de garbanzo, al no haber alternativa para el control de estas plagas a partir de la década de los 90 se dejaron de sembrar garbanzo en estas zonas.

De acuerdo con los boletines divulgativos de INIAP 1992, a partir de esta época el garbanzo que se consume en el país es importado de Perú, México y Canadá, por esa razón es necesario investigar sobre el comportamiento agronómico del cultivo de garbanzo en la zona tropical del país como una alternativa positiva agroecológica. Por lo tanto, nos planteamos determinar la adaptabilidad del garbanzo tropicalizado de origen peruano a las condiciones de suelo y temperatura de la zona de limoncito, Provincia de Santa Elena, que es parte zona costera del Ecuador.

\section{Materiales y métodos}

\section{Lugar de estudio}

El estudio se realizó durante el año 2018 a 2019 en El Limoncito, provincia de Santa Elena en época lluviosa y seca. Las condiciones edafoclimáticas son características de una zona tropical, con latitud de $2^{\circ} 13^{\prime} 0$ " latitud norte y longitud de $80^{\circ} 14^{\prime} 0$ " longitud oeste, con altitud $0 \mathrm{msnm}$, con una temperatura promedio de $28^{\circ} \mathrm{C}$ y una precipitación de $700 \mathrm{~mm}$.

\section{Diseño experimental}

La variedad del garbanzo (Cicer arietinum L.) con el que se trabajó en la presente investigación fue tipo kabuli de ascendencia peruana. Se realizó un diseño experimental de bloques completos al azar (DBCA) con cinco tratamientos y cuatro repeticiones. El ensayo fue dividido en parcelas y cada una correspondía a un bloque experimental, el área de cada parcela fue de $5 \mathrm{x} 5 \mathrm{~m}=25 \mathrm{~m}^{2} \mathrm{y}$ cada tratamiento correspondía a diferentes distanciamientos de siembra (Cuadro 1).

Cuadro 1. Distanciamientos de siembra de garbanzo por tratamiento.

\begin{tabular}{cc}
\hline Tratamiento & $\begin{array}{c}\text { Distanciamiento } \\
\text { (entre planta } \times \text { entre tratamiento, } \mathrm{m} \text { ) }\end{array}$ \\
\hline T1 & $0.1 \times 0.5$ \\
T2 & $0.2 \times 0.5$ \\
T3 & $0.3 \times 0.5$ \\
T4 & $0.4 \times 0.5$ \\
T5 & $0.5 \times 0.5$ \\
\hline
\end{tabular}

Para el desarrollo del ensayo se determinaron cinco tratamientos con cuatro repeticiones. Cada uno de ellos dependiendo su distanciamiento, variaba el número de semillas por parcela. En el T1 se sembró con 500 semillas, en el T2 con 250 semillas, en el T3 con 250 semillas, en el T4 con 250 semillas, mientras que en el T5 con el mayor distanciamiento de siembra con 100 semillas. Para determinar la adaptabilidad se determinaron las características agronómicas del cultivo a los 45 días, las cuales fueron: altura de planta, número de flores, número de ramas, número de vainas. 


\section{Rendimiento del garbanzo}

Después de la cosecha se procedió a recolectar 100 granos de garbanzo por planta y pesarlos, para luego determinar el rendimiento por planta en $\mathrm{kg} \mathrm{ha}^{-1}$.

\section{Análisis estadístico}

Para el análisis estadístico se utilizó el programa InfoStat. Los datos obtenidos fueron analizados mediante un análisis de varianza (Anova), luego con una prueba de significancia de Duncan, con $5 \%$ de probabilidad de error.

\section{Resultados y discusión}

De acuerdo con los análisis las plantas de garbanzo lograron un satisfactorio crecimiento y desarrollo fisiológico, evidenciados en las características agronómicas observadas.

\section{Determinación de la altura de planta}

La altura de planta fue medida cada 15 días hasta los 45 días, observándose un crecimiento normal y satisfactorio (Cuadro 2).

Cuadro 2. Promedio de altura de planta a los 45 días de sembrado.

\begin{tabular}{cl}
\hline Tratamientos & Altura de planta \\
\hline T1 & 25.03 A B C \\
T2 & 26.48 B C \\
T3 & $25.85 \quad$ C \\
T4 & $24.43 \quad$ A B \\
T5 & $23.35 \quad$ A \\
\hline
\end{tabular}

Medias con distinta letra, difieren estadísticamente (Duncan, $\alpha \leq 0.05$ ).

La altura de planta a los 45 días se indica en el Cuadro 2, donde el mejor resultado se observa en el tratamiento T2 con distanciamiento de siembra $0.2 \times 0.5 \mathrm{~m}$, el cual tiene como altura de planta $26.48 \mathrm{~cm}$.

En todos los tratamientos hubo diferencia significativa en cuanto a la altura de planta en donde el tratamiento T1 midió $24.03 \mathrm{~cm}$, el T3 con $25.85 \mathrm{~cm}$, T4 con $24.43 \mathrm{~cm}$ y el T5 con la menor altura de $23.35 \mathrm{~cm}$. Saluzzo (2010) indica que a los 60 días el garbanzo tiene como promedio de altura de planta $60 \mathrm{~cm}$.

La altura de planta está asociada con el crecimiento, el heliotropismo, el vigor competitivo, el tamaño reproductivo, la fecundidad de la planta, la longevidad promedio y la adaptabilidad del cultivo (Pérez et al., 2013). En Perú se estudiaron variedades de semillas de garbanzo condiciones de suelo de origen aluvial, en terreno plano y con pendiente de 0 a $1.5 \%$, suelos con drenaje moderado, donde el distanciamiento de siembra es de $80 \mathrm{~cm}$ por hilera y $40 \mathrm{~cm}$ por planta, con óptimos resultados (Chipana, 2015). 
Los distanciamientos de siembra analizados en investigaciones en México en zonas costeras determinan como recomendación que el distanciamiento del garbanzo por hilera sea de $80 \mathrm{~cm}$, este es un factor que a variar según las condiciones climáticas, el tipo de suelos, las condiciones topográficas y su fertilización apropiada para determinar el rendimiento y rentabilidad económica (Apáez et al., 2016).

\section{Número de flores por planta}

A los 45 días de siembra se contaron el número de flores (Cuadro 3) observándose buenos resultados en todos los tratamientos.

Cuadro 3. Promedio de número de flores por planta realizado a los 45 días.

\begin{tabular}{crl}
\hline Tratamientos & Número de flores por planta \\
\hline T1 & 92.25 & $\mathrm{~A} \mathrm{~B}$ \\
T2 & 93.5 & $\mathrm{~B}$ \\
T3 & 89.5 & $\mathrm{~A}$ \\
T4 & 105.25 & $\mathrm{~A} \mathrm{~B}$ \\
T5 & 102.5 & $\mathrm{C}$ \\
\hline
\end{tabular}

Medias con distinta letra, difieren estadísticamente (Duncan, $\alpha \leq 0.05$ ).

Según el análisis estadístico existen diferencias significativas entre los tratamientos, evidenciándose que el distanciamiento de siembra influye en el desarrollo de la planta. Los tratamientos T4 y T5 fueron los de mayor numero de flores con 105 y 102 respectivamente, mientras que el T3 es el de menor número de flores con 89 y los tratamientos T1 y T2 con 92 y 93 respectivamente.

\section{Número de ramas por planta}

El promedio de número de ramas por planta a los 45 días de todos los tratamientos es de 4 , a diferencia del T2 el cual tubo cinco ramas, observándose que no hubo diferencias significativas, según el análisis estadístico.

\section{Número de vainas por planta}

En el número de vainas por planta a los 45 días (Cuadro 4), asimismo, no tubo diferencias significativas, teniendo un promedio en los tratamientos T1, T2, T3 40 vainas, mientras que los T4 y T5 se observaron 39 vainas.

Cuadro 4. Promedio de número de vainas por planta a los 45 días.

\begin{tabular}{cc}
\hline Tratamientos & Número de vainas por planta \\
\hline T1 & 40 \\
T2 & 40 \\
T3 & 40 \\
T4 & 39 \\
T5 & 39 \\
\hline
\end{tabular}




\section{Determinación del peso de 100 granos de garbanzo}

Después de tres meses de cosecha se procedió a recolectar los 100 granos de garbanzo y pesarlos. En los resultados de análisis estadísticos observa que existe diferencia significativa entre los ensayos realizados (Cuadro 5). Se localizó que los tratamientos T1 y T2 con un promedio de 48.46 g y 49.83 g respectivamente fueron los de mayor peso. El garbanzo tipo kabuli presentó un peso de 100 semillas mayor a 25 gramos, mientras que las 100 semillas del tipo desi pesan menos de 25 gramos (Vizgarra et al., 2017).

Cuadro 5. Peso de 100 granos de garbanzo.

\begin{tabular}{ccccc}
\hline Tratamiento & \multicolumn{3}{c}{ Peso $(\mathrm{g})$} \\
\hline T1 & 48.46 & A & & \\
T2 & 49.83 & A & & \\
T3 & 34.22 & & B & \\
T4 & 33.16 & B & & \\
T5 & 29.48 & & C & \\
\hline
\end{tabular}

Medias con distinta letra, difieren estadísticamente (Duncan, $\alpha \leq 0.05$ ).

\section{Rendimiento}

De acuerdo con los promedios obtenidos en cuanto a la variable rendimiento expresado en $\mathrm{kg} \mathrm{ha}^{-1}$ (Cuadro 6), se observó que el mejor distanciamiento de siembra corresponde al tratamiento T2 con un promedio de $1814.14 \mathrm{~kg} \mathrm{ha}^{-1} \mathrm{y}$ el de menor tratamiento de $986.03 \mathrm{~kg} \mathrm{ha}^{-1}$, lo cual concuerda con los resultados de ensayos realizados en la ciudad de México (Moral et al., 1994).

Cuadro 6. Promedio del rendimiento en $\mathrm{kg} \mathrm{ha}^{-1}$.

\begin{tabular}{ccc}
\hline Tratamientos & Rendimiento $\left(\mathrm{kg} \mathrm{ha}^{-1}\right)$ & \\
\hline T1 & 1766.53 & $\mathrm{~A}$ \\
T2 & 1814.14 & $\mathrm{~A}$ \\
T3 & 1106.48 & $\mathrm{~B}$ \\
T4 & 1088.3 & $\mathrm{~B}$ \\
T5 & 986.03 & $\mathrm{C}$ \\
\hline
\end{tabular}

Medias con distinta letra, difieren estadísticamente (Duncan, $\alpha \leq 0.05$ ).

De los diferentes rendimientos en los diferentes distanciamientos de siembra evaluados (Figura 1) en el presente ensayo se puede concluir que el tratamiento que tuvo el mejor comportamiento agronómico fue el tratamiento $\mathrm{T} 2(0.2 \times 0.5 \mathrm{~m})$ con $1814.14 \mathrm{~kg} \mathrm{ha}^{-1} \mathrm{y}$ el tratamiento que tuvo el menor rendimiento fue el tratamiento T5 con $986.03 \mathrm{~kg} \mathrm{ha}^{-1}$ lo cual demuestra que para que las condiciones edafoclimáticas de Limoncito el distanciamiento de $0.2 \times 0.5 \mathrm{~m}$ es el tratamiento con mayor rendimiento. 


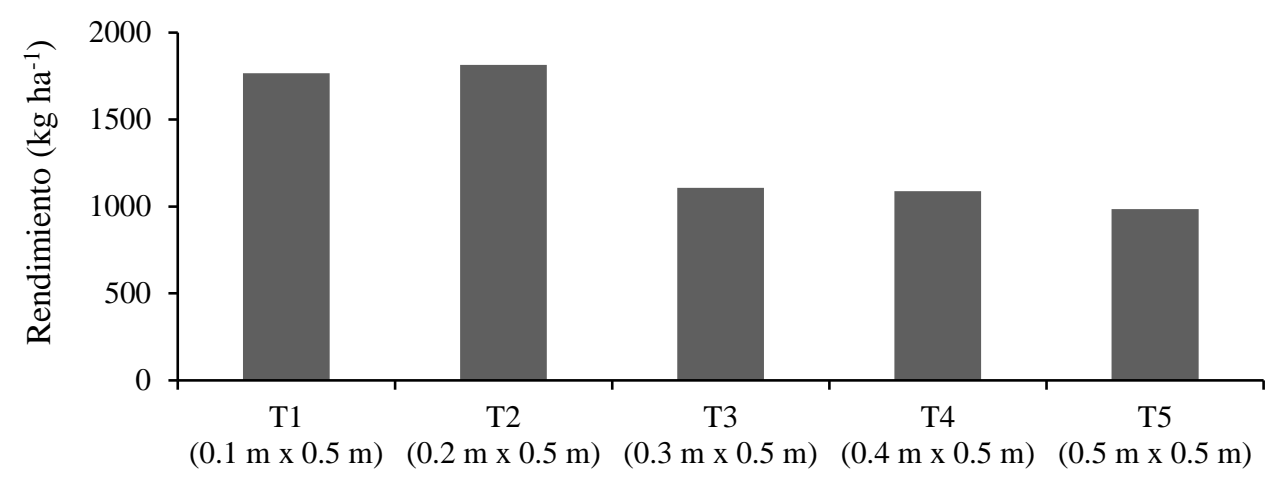

Figura 1. Representación gráfica en barras del rendimiento de garbanzo.

\section{Conclusiones}

La presente investigación demuestra que la adaptabilidad del garbanzo tropicalizado de ascendencia peruana, tipo kabuli, en las condiciones edafoclimáticas de El Limoncito, Ecuador es muy favorable, observándose que el T2 con un distanciamiento de siembre de 0.2 x $0.5 \mathrm{~m}$ es el de mejor desarrollo agronómico de la planta en cuanto a la altura de planta, numero de flores, numero de ramas, numero de vainas. Esto plantea una buena opción para el agricultor de zonas tropicales como alternativa de producción de cultivos.

\section{Agradecimientos}

Los autores agradecen al Instituto de Investigación e Innovación (SINDE) de la Universidad Católica de Santiago de Guayaquil (UCSG), por el financiamiento del proyecto: 'Adaptación del garbanzo (Cicer arietinum) a las condiciones edafoclimáticas de El Limoncito, provincia de Santa Elena', que es parte del presente artículo.

\section{Literatura citada}

Abi-Ghanem, R.; Carpenter-Boggs, L.; Smith, J.; Vandemark, G. 2012. Nitrogen fixation by US and middle eastern chickpeas with commercial and wild middle eastern inocula. ISRN Soil Science.1-5 pp.

Anderson, J. T.; Willis, J. H. and Mitchell-Olds, T. 2011. Evolutionary genetics of plant adaptation. Trends in genetics: TIG. 27(7):258-266.

Apáez, B. M.; Escalante, E. J. A. S.; Rodríguez, G. M. T.; Sosa, M. E. y Apáez, B. P. 2016. Distancia entre hileras, nitrógeno y producción de garbanzo en humedad residual. Rev. Mex. Cienc. Agríc. 7(2):223-234.

Carranca, C. de V. A. and Rolston, D. 1999. Biological nitrogen fixation by fababean, pea and chickpea, under field conditions, estimated by the isotope dilution technique. European Journal of Agronomy. 10(1):49-56.

Chipana, C. 2015. Ensayo de treinta variedades de garbanzo (Cicer arietinum L.) sembrado en invierno para condiciones de costa central (Tesis de grado). UNA La Molina Lima, Perú.

Coirini, A. y Nolasco, M. 2016. El cultivo de garbanzo en Córdoba: análisis con imágenes satelitales. CAI, $8^{\circ}$ Congreso de AgroInformática. 67-80 pp. 
FAO. Organización de las Naciones Unidas para la Alimentación y la Agricultura. 1997. The state of the world's plant genetic resources for food and agriculture. FAO: Roma. Obtenido de: http://www.fao.org/3/a-w7324e.pdf)\%D8\%9B.

FAO. Organización de las Naciones Unidas para la Alimentación y la Agricultura. 2020. FAOSTAT. Obtenido de: http://www.fao.org/faostat/en/\#data/QC.

Fierros, G.; Ortega, P.; Acosta, J.; Padilla, I.; Valenzuela, V.; Jiménez, Y. y López, J. 2011. Respuesta del rendimiento de genotipos de garbanzo blanco a la sequía terminal. Rev. Mex. Cienc. Agríc. 8(5):1143-1154.

Heidarvand, L. and Maali-Amiri, R. 2013. Physio-biochemical and proteome analysis of chickpea in early phases of cold stress. J. PlantPhysiol. 170:459-469.

Kazydub, N.; Kuzmina, S. and Chernenko, S. 2017. Adaptability of chickpea collection samples in the southern forest-steppe of western Siberia. Bulgarian Journal of Agricultural Science. 23(5):743-749.

Kirnak, H.; Varol, I.; Irik, H. and Ozaktan, H. 2017. Effects of Irrigation Applied at Different Growth Stages on Chickpea Yield. Agronomy Research. 15(5):1928-1933.

Moral, J.; Mejias, A. y López, M. 1994. El cultivo del garbanzo: diseño para una agricultura sostenible. Madrid Ministerio de Agricultura Pesca y Alimentación, Secretaria General Técnica. Hojas divulgadoras. España. Ministerio de Agricultura, Pesca y Alimentación. 12-94 pp.

Nisa, Z.; Arif, A. and Waheed, M. Q. et al. 2020. A comparative metabolomic study on desi and kabuli chickpea (Cicer arietinum L.) genotypes under rainfed and irrigated field conditions. Sci. Rep. 10:13919.

Pérez, N.; Díaz, S.; Garnier, E.; Lavorel, S.; Poorter, H.; Jaureguiberry, P.; Bret-Harte, M.; Cornwell, W.; Craine, J.; Gurvich, D.; Urcelay, C.; Veneklaas, E.; Reich, P.; Poorter, L.; Wright, I.; Ray, P.; Enrico, L.; Pausas, J.; de Vos, A.; Buchmann, N.; Funes, G.; Quétier, F.; Hodgson, J.; Thompson, K.; Morgan, H. D.; Ter Steege, H.; Van der Heijden, M.; Sack, L.; Blonder, B.; Poschlod, P.; Vaieretti, M.; Conti, G.; Staver, A.; Aquino, S. and Cornelissen, J. 2013. New handbook for standardised measurement of plant functional traits worldwide. Australian Journal of Botany. 61:167-234.

Saluzzo, A. 2010. Adaptación del cultivo de garbanzo en función de la variabilidad ambiental. $3^{\text {a }}$ Jornada Nacional de Garbanzo, INTA EEA Salta.

Shagarodsky, T.; Chiang, M. y López, Y. 2001. Evaluación de cultivares de garbanzo (Cicer arietinum 1.) en Cuba. Agronomía Mesoamericana. 12(1):95-98.

Sharma, R. and Johnson, P. 2017. Genotype x environment interaction and stability analysis for yield traits in chickpea (Cicer arietinum L.). Electronic Journal of Plant Breeding. 8(3):865-869.

Sharma, R.; Abraham, S.; Bhagat, R.; Mishra, T. and Prakash, O. 2018. Evaluation of chickpea varieties treated with bio inoculants for yield performance, disease resistance and adaptability to climatic conditions of Gariyaband district in Chhattisgarh. Legume Research. 41(1):57-59.

Vizgarra, O.; Espeche, C.; Silvana, Y.; Mamaní, G. y Daniel, P. L. 2017. TUC 403 y TUC 464, dos nuevas variedades de garbanzo tipo Kabuli para el noroeste argentino. Rev. Ind. y Agríc. de Tucumán. 94(1):41-47.

Wood, J. and Grusak, M. 2007. Nutritional Value of Chickpea. In Chickpea Breeding and Management. CAB International. 101-142 pp. 